\title{
Quality of life in children and adolescents with obsessive-compulsive disorder: a systematic review and meta-analysis
}

This article was published in the following Dove Press journal:

Neuropsychiatric Disease and Treatment

24 February 2017

Number of times this article has been viewed

\section{Anna Coluccia' \\ Fabio Ferretti ${ }^{1}$ \\ Andrea Fagiolini ${ }^{2,3}$ \\ Andrea Pozza'}

'Department of Medical Sciences, Surgery and Neurosciences, Santa Maria alle Scotte University Hospital, ${ }^{2}$ Department of Molecular Medicine, School of Medicine, University of Siena, ${ }^{3}$ Department of Mental Health, University of Siena Medical Center (AOUS), Siena, Italy
Correspondence: Anna Coluccia Department of Medical Sciences, Surgery and Neurosciences, Santa Maria alle Scotte University Hospital, Viale Bracci 16, 53 100 Siena, Italy Tel +39057758 6409

Fax +39057723 3222

Email coluccia@unisi.it
Abstract: Obsessive-compulsive disorder (OCD) is a seriously impairing psychiatric condition that affects $1 \%-3 \%$ of youth. Investigating the quality of life (QOL) is an important issue for treatment planning of this disorder, as targeting symptoms without taking it into account may bias assessment and prognosis when the patient presents with reduced symptoms that do not correspond to improved QOL. However, QOL in young individuals with OCD has been understudied. This meta-analysis summarized current evidence that assessed differences in global, social and school QOL dimensions, between children/adolescents with OCD and screened controls. Age, sex and OCD severity were examined as moderators. Case-control studies were included if children/adolescents with primary OCD were compared with screened controls on validated self-reported QOL outcomes. Online databases (January 1966-January 2016) were searched. Five case-control studies were included ( $\mathrm{n}=543,17$ effect sizes overall). On global QOL, a large effect size emerged $(d=-1.16, P<0.001)$, suggesting that individuals with OCD had lower global QOL than controls. Moderate effect sizes emerged for school $(d=-0.61, P<0.01)$ and social QOL ( $d=-0.54, P<0.01)$, respectively, indicating worse QOL on these domains for individuals with OCD. For samples with higher OCD severity, global QOL of individuals with OCD was lower than that for controls $(\beta=-0.02, P<0.05)$. For samples with lower percentages of females, global QOL of individuals with OCD was more impaired $(\beta=0.02, P<0.001)$. Age was not correlated with effect sizes. Assessment and treatment should target QOL for young males suffering from more severe OCD. The small number of included studies highlighted that QOL is under-recognized. Future research should focus on additional QOL domains and compare which ones are impaired among individuals with OCD compared with other psychiatric conditions. Overall, the results pointed out the importance of addressing QOL in both practice and research on assessment and treatment of children/adolescents with this condition.

Keywords: obsessive-compulsive disorder, youth, functioning, social quality of life, severity, case-control study

\section{Introduction}

Obsessive-compulsive disorder (OCD) in children and adolescents is a psychiatric disease consisting of unwanted, intrusive thoughts or mental images (obsessions) and repetitive behaviors or mental acts (compulsions) that are engaged to reduce discomfort created by obsessions. ${ }^{1}$ Natural course is chronic and has a first peak around puberty and another in early adulthood. ${ }^{2}$ While some studies suggested that age of onset is earlier in boys than in girls, ${ }^{3}$ other studies found comparable ages of onset. ${ }^{4}$ Symptoms affect $\sim 1 \%-3 \%$ of young individuals, are time-consuming and significantly interfere with quality of life (QOL). ${ }^{4,5}$ In addition to OCD, up to $75 \%$ of young patients also 
meet criteria for one or more comorbid disorders, with the most frequent being depressive disorders, anxiety disorders, attention-deficit/hyperactivity disorder (ADHD) and disruptive behavior disorders. ${ }^{5,6}$ Moreover, many young people suffering from OCD experience difficult peer relationships and school difficulties and participate in fewer recreational activities than non-affected peers. ${ }^{7.8}$

Following some authors, QOL can be defined as "the perception of the impact of disease on a variety of dimensions including physical, mental, and social domains". Investigating QOL in young individuals with OCD has some implications for assessment and treatment. For the clinician performing assessment, knowledge of the patient's perceptions of QOL provides important information as a subjective estimate of his/her overall functioning with regard to prognosis. ${ }^{8-10}$ In addition, targeting OCD symptoms without considering QOL of the patient may bias assessment as he/she may present with reduced symptoms that do not correspond to improved daily functioning. ${ }^{8-10}$ With regard to treatment, despite the substantial impairments in QOL associated with $\mathrm{OCD}$, treatment outcome evaluations have focused almost exclusively on symptom reduction; however, focusing on symptom remission may not help the clinician to understand whether a clinically significant change has occurred. ${ }^{8-10}$ In line with these arguments, symptom improvement observed on standardized measures did not always correspond to clinically significant changes in real-world QOL and functioning. ${ }^{8-11}$ Moreover, other studies have shown that limited symptom gains were associated with significant $\mathrm{QOL}$ decreases, or not improved QOL at the end of treatment was associated with higher risk for relapse..$^{8-11}$

Studying patients' perceptions of QOL appears a relevant issue particularly for clinical practice with young individuals, as most of the literature indicated that OCD among children/ adolescents was mainly characterized by the absence of insight of symptoms and the associated impairment. ${ }^{5,6}$ Thus, OCD severity and QOL could not have a direct relation. In groups of individuals with OCD, some recent studies ${ }^{12}$ found that OCD severity was not significantly correlated with QOL. On the other hand, other studies ${ }^{13}$ showed that severity of obsessions significantly predicted perceived QOL, whereas severity of compulsions did not impact on QOL ratings. In contrast, a growing body of evidence showed that adult individuals with OCD experienced lower QOL relative to healthy controls and an inverse relation between QOL and OCD symptom severity, suggesting that individuals with less severe OCD had poorer QOL than those with more severe symptoms. ${ }^{14,15}$
While a great body of data have been accumulated on QOL among adults, ${ }^{15}$ this topic among youth is underrecognized and little empirical work has been conducted. ${ }^{7,8}$ This aspect appears important as most of the studies indicated that the juvenile form of the disorder may present relevant clinical differences compared with the adult counterpart in terms of severity, comorbidities, insight of symptoms and treatment response. ${ }^{6}$

Therefore, this systematic review and meta-analysis summarized available evidence looking for differences in global QOL outcomes between children/adolescents with OCD and screened healthy controls. In addition, specific QOL dimensions were examined, in particular social and school-related QOL. Finally, the effects of OCD symptom severity, sex and age were investigated. These variables were investigated as moderators, based on previous findings from the literature. Previous research on adults suggested that sex had a crucial role in moderating the effects of OCD on QOL; specifically, female sex was associated with lower QOL. ${ }^{15}$ Other data on youth instead found that male individuals with OCD had more severe characteristics, including earlier age of onset and poorer QOL. ${ }^{16}$ With regard to age, inconsistent evidence has been found, with some studies showing differences in the clinical picture between children and adolescents and others not. ${ }^{3,6}$

\section{Methods \\ Eligibility criteria}

Following a revised version of the PICOS method for observational case-control studies, defined in the PRISMA guidelines, ${ }^{17}$ criteria for inclusion of the studies involved the following characteristics: 1) characteristics of participants. Studies were included if participants were children or adolescents with a primary diagnosis of OCD. Age range for children was defined as between 6 and 14 years, whereas adolescence age range was considered as $15-18$ years. Diagnosis had to be made through a semi-structured interview based on standardized diagnostic criteria, such as the Schedule for Affective Disorders and Schizophrenia for school-age children - present and lifetime version (K-SADS-PL).$^{18}$ Thus, studies on individuals with subclinical OCD symptoms were excluded. Studies were also excluded if they were conducted on individuals with the pediatric autoimmune neuropsychiatric disorders associated with streptococcal infections (PANDAS), as there are some important clinical differences between OCD and this form of neurological condition. ${ }^{19}$ Studies on primary compulsive hoarding were excluded, since this condition has been included as a separate diagnosis 
in the Diagnostic and Statistical Manual of Mental Disordersfifth edition (DSM-5). ${ }^{1}$ 2) Characteristics of outcomes. Studies were included if they used self-reported measures of global, social and school QOL outcomes with known psychometric properties. 3) Characteristics of comparators. Studies were included if they used healthy control groups consisting of screened participants who have not reported any lifetime psychiatric disorder during a clinical interview.

4) Characteristics of design. Studies were included if they used an observational case-control design, where groups of individuals with a primary OCD diagnosis were compared with healthy control groups.

\section{Information sources and search procedure}

Studies were retrieved through electronic systematic literature searches, in which keywords related to OCD ("obsessive compulsive disorder", "obsessions", "compulsions", "rituals") were combined through the Boolean operator "AND" with keywords and text words indicative of the QOL construct ("quality of life", "psychological well-being", "satisfaction with life", "global functioning", "social functioning", "family functioning", "subjective well-being"). The following online databases were searched: PubMed (January 1966-January 2016), ScienceDirect (January 1966-January 2016) and PsycINFO (January 1966-January 2016).

\section{Process of selection of the studies}

Studies were assessed on eligibility criteria by three independent reviewers (FF, AP and AC) during three different stages. During the first and the second one, studies were examined with regard to inclusion criteria after the reading of the title and of the abstract, respectively. During these stages, studies were retained when there was no agreement on inclusion between the reviewers. Finally, the remaining studies were assessed on eligibility criteria after reading the full text of the article. At this stage, reasons for inclusion/exclusion and potential discrepancy in the judgment were addressed during meetings with a fourth independent reviewer (AF) to obtain complete agreement on included studies for meta-analysis.

\section{Moderators coding}

When inconsistency analyses indicated large and significant heterogeneity between the effect sizes, the role of moderators was investigated. Two independent reviewers (FF and AP) coded the moderators, extracted the data from primary studies and inserted them in an excel worksheet. Subsequently, during meetings between the two reviewers, insertion of the data in the worksheets was checked for accuracy, and each potential discrepancy was discussed and resolved with a third reviewer (AC). Discrepancy was found for coding of mean age and percentage of females/males for two studies. It was discussed by the two reviewers and resolved through the check of the papers by the third reviewer. The following variables were coded as moderators: 1) mean age of the sample; 2) sex of the sample, coded as percentage of female participants and 3) OCD symptom severity, coded as a continuous variable based on the scores on the Children's Yale-Brown Obsessive Compulsive Scale (CY-BOCS). ${ }^{20}$

\section{Meta-analysis}

\section{Data extraction and summary measures}

As we expected noticeable heterogeneity across the included studies, the effect sizes were calculated using random-effect models. Data requested for the calculation of the effect sizes were extracted independently by two meta-analysts (FF and AP) through the following formula: $E S=M_{O C D}-M_{C O N T R O L}$ $S D_{\text {POOLED }}$, where $M_{O C D}$ was the mean of groups with OCD, $M_{\text {CONTROL }}$ was the mean of controls and $S D_{\text {POOLED }}$ was the pooled standard deviation. ${ }^{21}$ The effect sizes were weighted through the following correction: $W_{\mathrm{zr}}=1 / S E_{\mathrm{zr}}^{2}$, where $S E_{\mathrm{zr}}^{2}$ was the standard error of the effect size computed for each study. This formula was further corrected through the following formula for sampling error: $W^{\prime}=W_{\mathrm{zr}} \cdot\left(\alpha_{\mathrm{QOL}}\right) \cdot\left(\alpha_{\mathrm{OCD}}\right)$, where $\alpha_{\mathrm{QOL}}$ was the Cronbach's coefficient ${ }^{22}$ as internal consistency for the QOL measures and $\alpha_{\mathrm{OCD}}$ was the coefficient for OCD measures. Effect sizes were estimated using a 95\% confidence interval $(95 \% \mathrm{CI})$ and interpreted according to the criteria by Cohen. ${ }^{21}$ Thus, effect sizes of 0.80 or more were assumed to be large, 0.50 moderate and 0.20 small. A global effect size was calculated as a mean effect size obtained by combining effect sizes related to different QOL measures. For those studies using QOL measures related to different QOL domains (eg, social and school QOL), a mean effect size was calculated for each study as a global QOL outcome. Subsequently, effect sizes were computed separately for specific domains, social and school QOL, respectively.

\section{Inconsistency analysis}

In order to assess between-studies heterogeneity, two complementary indices are used, the $I^{2}$ index and the $Q$-statistic, respectively. A value of $I^{2}$ approximating to zero suggests homogeneity, whereas values of $25 \%-50 \%, 50 \%-75 \%$ and $75 \%-100 \%$ represented low, medium and large heterogeneity, respectively. ${ }^{23}$ Given that inconsistency analysis suggested large heterogeneity, an analysis of the above-mentioned 
moderators was conducted through weighted least squares meta-regressions.

\section{Publication bias}

In order to investigate the likelihood that effect sizes were subjected to publication bias, the trim and fill procedure of Duval and Tweedie ${ }^{24}$ and the visual inspection of the funnel plot were used. The meta-analysis was performed using the Comprehensive Meta-analysis software, version 2.00.

\section{Results}

\section{Selection and descriptive characteristics of the studies}

The electronic search and the search through additional sources produced 1,067 records after the removal of duplicates. Of those studies, 1,005 were excluded at title or at abstract, as they were on irrelevant constructs. Thus, 62 studies were screened at full text for inclusion. Of those studies, nine studies were excluded as they did not report sufficient data to calculate effect sizes (eg, standard deviations), and the authors did not reply when they were contacted to request for necessary data. Two studies were excluded as they did not use measures of QOL. Twenty-three studies were excluded as they did not use a case-control design (eg, the study did not use a control group). Three studies were excluded as they did not use validated QOL measures. Two studies were excluded as they used participants with lifetime OCD diagnosis. One study was excluded as it used participants with subclinical OCD symptoms. One study was excluded as it used participants with hoarding, and one study was excluded as it used the same data as those reported in a study already included in the meta-analysis. Sixteen studies were excluded since they were conducted on adult samples. After this selection, five studies were included in both qualitative and quantitative synthesis ( $n=543,17$ effect sizes overall) by consensus of the three independent assessors. The flowchart of the selection process is provided in Figure 1.

All the included studies compared QOL outcomes of patients with OCD and screened healthy participants as controls. Participants in the OCD groups were outpatients in all the studies. Three studies were conducted in the United States, ${ }^{6,25,26}$ one in Brazil, ${ }^{27}$ and one in Argentina. ${ }^{28}$ Total sample sizes ranged from 51 to 225 participants. One study was conducted on adolescents ${ }^{27}$ and four on children. ${ }^{6,25,26,28}$ Mean percentage of females was 51.70 ranging from 44.60 in the study of Geller et $a l^{6}$ to 66.20 in the study of Sousa Vivan et al. ${ }^{27}$ With regard to comorbidities, in the study of Borda et al, ${ }^{28}$ psychiatric comorbidity included dysthymia (10\% of the clinical group), specific phobias (10\%), separation anxiety (10\%), panic disorder with agoraphobia (4\%), eating disorders (10\%) and selective mutism (4\%). In the study of Sukhodolsky et al, ${ }^{26} 56$ individuals had OCD and 43 had OCD with comorbid ADHD. In the study of Sukhodolsky et al, ${ }^{26}$

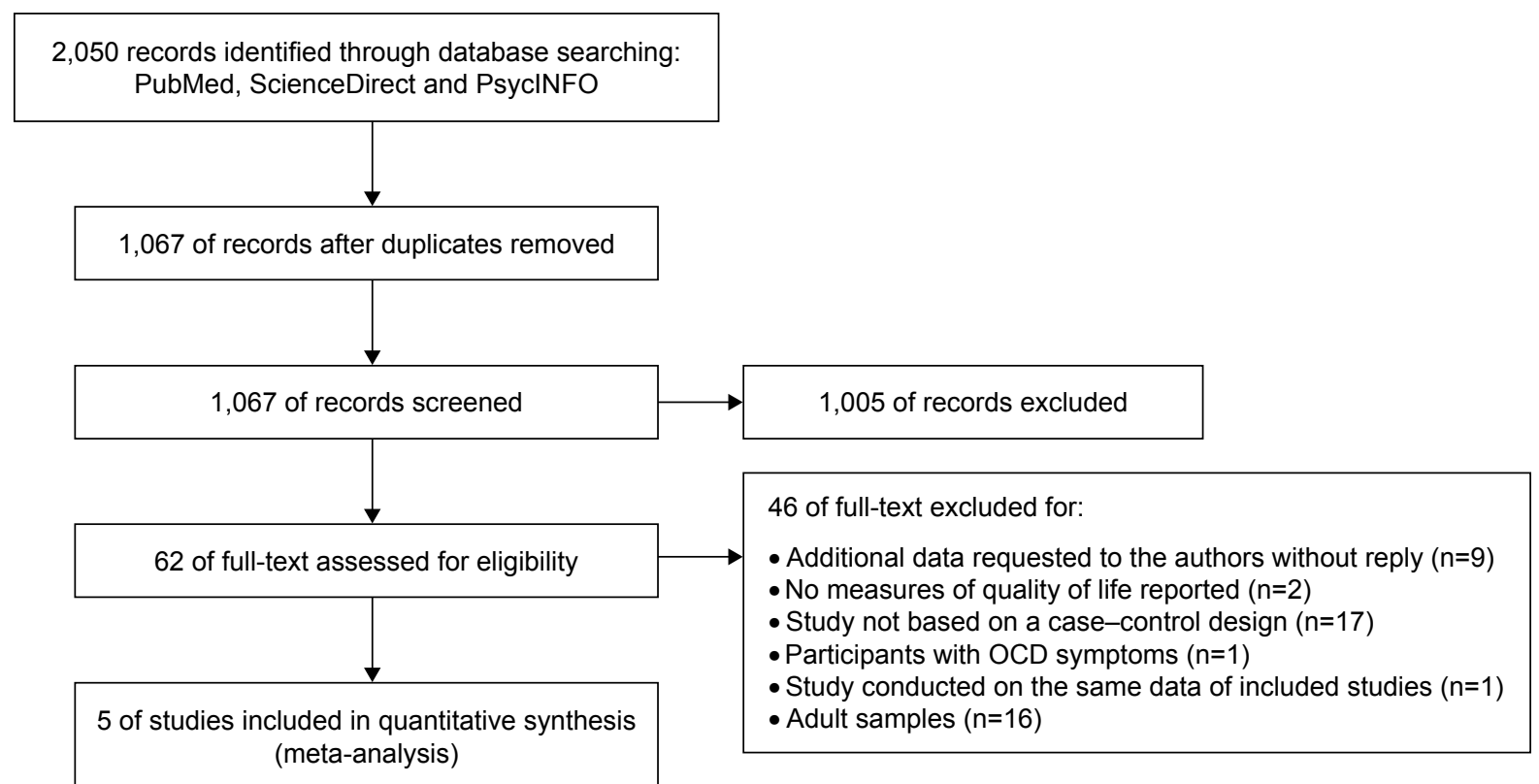

Figure I PRISMA flowchart of the selection process of the studies. Abbreviation: OCD, obsessive-compulsive disorder. 
$41 \%$ had comorbid Tourette's disorder, $28 \%$ had comorbid depression, $41 \%$ had comorbid anxiety disorders and 13\% had comorbid oppositional defiant disorder. In the study of Kim et al, ${ }^{25} \sim 45 \%$ had a comorbid generalized anxiety disorder, $8 \%$ had comorbid social anxiety disorder, $12 \%$ had specific phobias, $8 \%$ had separation anxiety disorder, $4 \%$ had an anxiety disorder not otherwise specified, $8 \%$ had major depression, 4\% had dysthymia, 33\% had ADHD, 8\% had Tourette's disorder, 12\% had oppositional defiant disorder and $4 \%$ had agoraphobia.

Two studies ${ }^{6,27}$ did not report data on comorbidities. Three studies reported scores on the CY-BOCS: mean score was 18.53 ranging from 11.60 in the study of Sukhodolsky et $\mathrm{al}^{26}$ to 22.90 in the study of Borda et al. ${ }^{28}$

In the study of Borda et al, ${ }^{28}$ OCD subtypes were reported. The most frequent obsessions included somatic (43\%), contamination (39\%), aggressive $(30 \%)$, symmetry $(26 \%)$, religious (9\%) and sexual obsessions (4\%); 48\% also reported a variety of additional miscellaneous obsessions (ie, need to know and remember, fear of saying certain things, fear of not saying the right thing, intrusive images and intrusive sounds, words or music). Similarly, a range of compulsions were reported, including cleaning (52\%), repeating (52\%), ordering (43\%), checking (35\%), counting (22\%) and other mental compulsions (13\%); $25 \%$ also reported a variety of additional miscellaneous compulsions (ie, excessive list utilization, superstitious behaviors).

Only one study reported whether the participants in the OCD groups were on medication. ${ }^{26}$ Two studies used patient-reported measures of QOL, ${ }^{27,28}$ whereas three used parent-reported measures. ${ }^{6,25,26}$ Publication date ranged from 1996 to 2014. Characteristics for each of the included studies are presented in Table 1.

\section{Comparison on global QOL between individuals with OCD and controls}

This analysis included five studies $(n=543)$. Results showed a large effect size $(d=-1.16, \mathrm{SE}=0.32,95 \% \mathrm{CI}:-1.79$ to 0.54 , $P<0.001$ ), suggesting that patients with OCD had significantly lower scores on global QOL outcomes compared with controls. A large and significant heterogeneity was found $\left(I^{2}=89.07, Q_{(4)}=36.61, P<0.001\right)$. The forest plot with study and mean effect sizes is presented in Figure 2.

Evidence for a publication bias was not found since the effect size did not change when it was adjusted for publication bias ( $d=-1.16$; $95 \% \mathrm{CI}:-1.78$ to 0.54 ; number of trimmed studies $=0$ ). Funnel plot of publication bias is presented in Figure 3.

\section{Analysis of moderators}

Since significant heterogeneity was found across effect sizes of the studies on global QOL, age, sex and OCD symptom severity were assessed as moderators. OCD symptom severity resulted to be negatively correlated with effect sizes $(\beta=-0.02, \mathrm{SE}=0.01,95 \% \mathrm{CI}:-0.05$ to $0.001, P<0.05)$, suggesting that for samples with more severe OCD symptoms, global QOL of patients was significantly lower than that for samples of controls. Meta-regression of global QOL as a function of OCD severity is presented in Figure 4.

Age was not correlated with effect sizes $(\beta=0.001$, $\mathrm{SE}=0.02,95 \% \mathrm{CI}:-0.03$ to $0.03, P=0.99)$. Meta-regression of global QOL as a function of age is presented in Figure 5. Sex was correlated with effect sizes $(\beta=0.02, \mathrm{SE}=0.01$, 95\% CI: $0.01-0.02, P<0.001)$, suggesting that for samples with higher percentages of males, global QOL of patients with OCD was significantly higher. Meta-regression of global QOL as a function of sex is presented in Figure 6. A summary of analysis of moderators is presented in Table 2.

\section{Comparison on social QOL between patients with OCD and controls}

This analysis included four studies $(n=490)$. Results showed a moderate effect size $(d=-0.54, \mathrm{SE}=0.15,95 \% \mathrm{CI}$ : -0.85 to $0.23, P<0.01$ ), suggesting that patients with OCD had significantly lower scores on social QOL compared with controls. Evidence for heterogeneity was not found $\left(I^{2}=56.51\right.$, $\left.Q_{(3)}=6.89, P=0.08\right)$. The forest plot with study and mean effect sizes is presented in Figure 7. Evidence for a publication bias was not found since the effect size did not change when it was adjusted for publication bias $(d=-0.54 ; 95 \%$ CI: -0.84 to 0.23 ; number of trimmed studies $=0$ ). Funnel plot is presented in Figure 8.

\section{Comparison on QOL related to school functioning between patients with OCD and controls}

This analysis included three studies $(\mathrm{n}=265)$. Results showed a moderate effect size $(d=-0.61, \mathrm{SE}=0.13,95 \% \mathrm{CI}$ : -0.87 to $0.36, P<0.01)$, suggesting that patients with OCD had significantly lower scores on school QOL outcomes compared with controls. Evidence for heterogeneity was not found $\left(I^{2}=1.89, Q_{(2)}=2.04, P=0.36\right)$. The forest plot is presented in Figure 9. Evidence for a publication bias was not found since the effect size did not change when adjusted for publication bias ( $d=-0.61 ; 95 \% \mathrm{CI}$ : -0.87 to 0.36 ; number of trimmed studies $=0$ ). Funnel plot of publication bias is presented in 


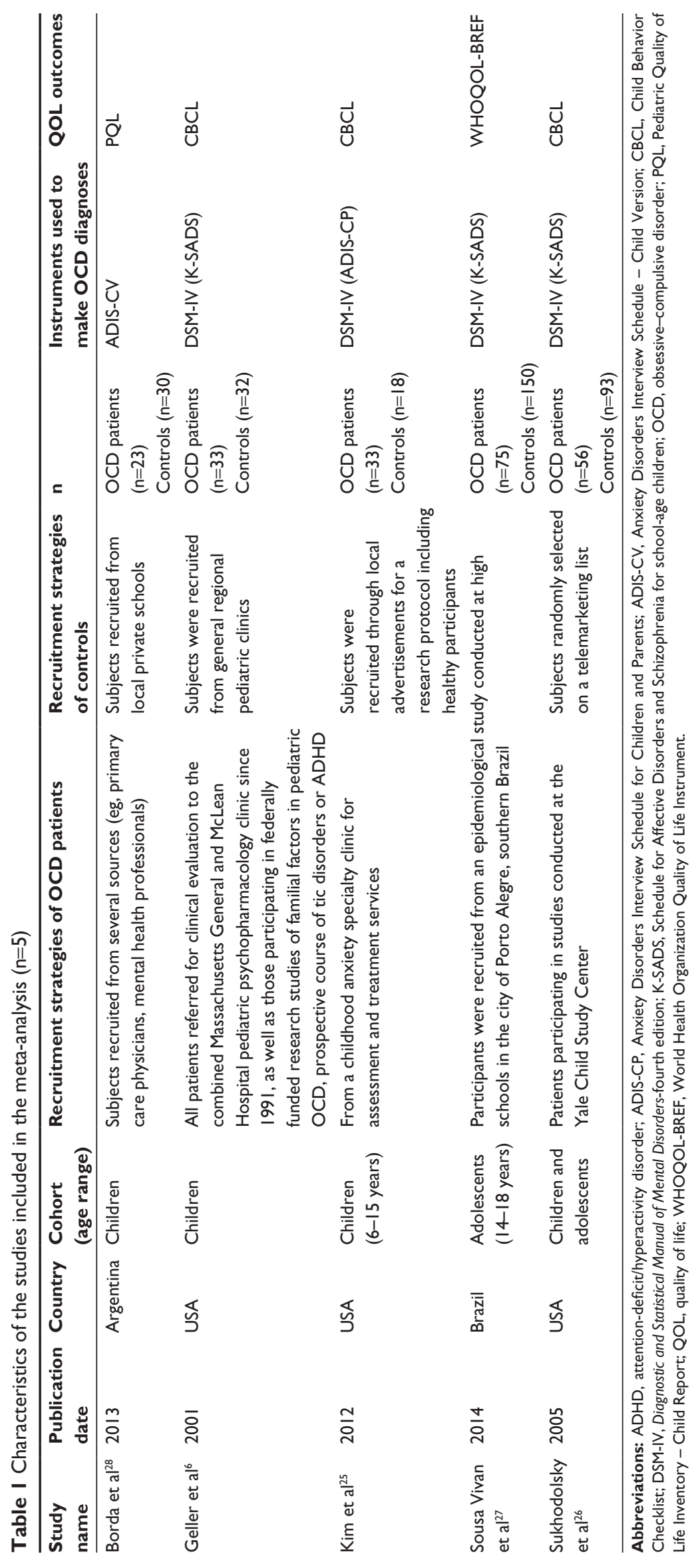




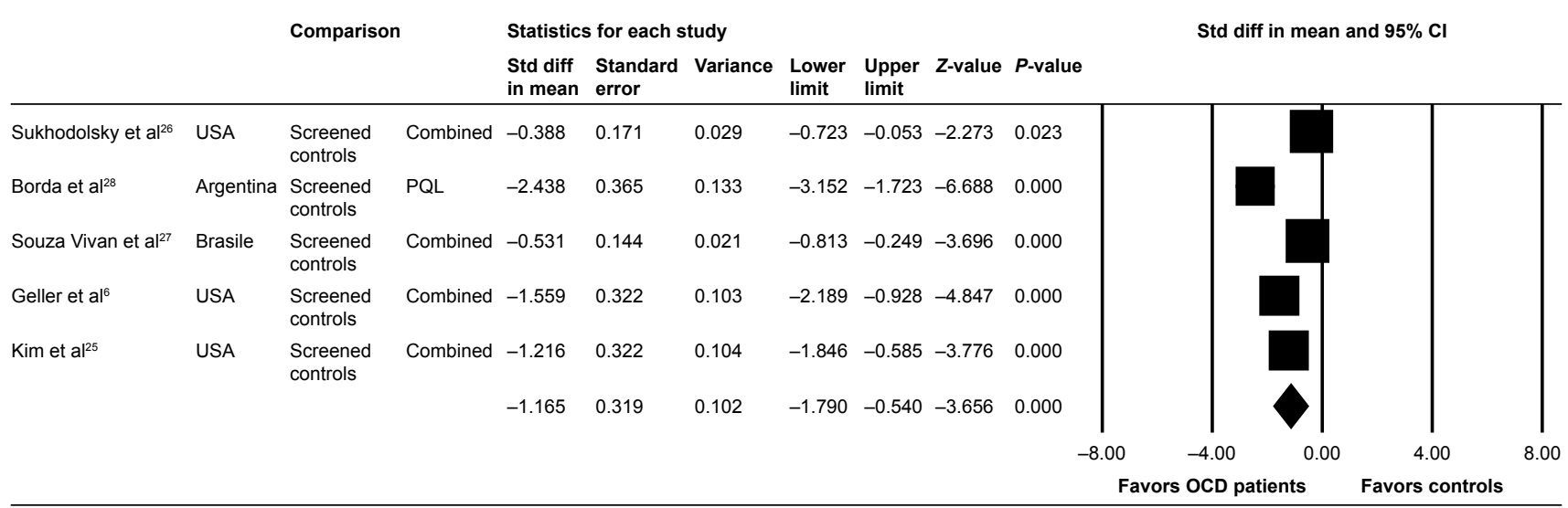

Meta-analysis

Figure 2 Forest plot of the comparison on global $Q O L$ between individuals with $O C D$ and controls.

Abbreviations: QOL, quality of life; OCD, obsessive-compulsive disorder; Std diff, standard difference; $\mathrm{Cl}$, confidence interval; PQL, Pediatric Quality of Life Inventory Child Report.

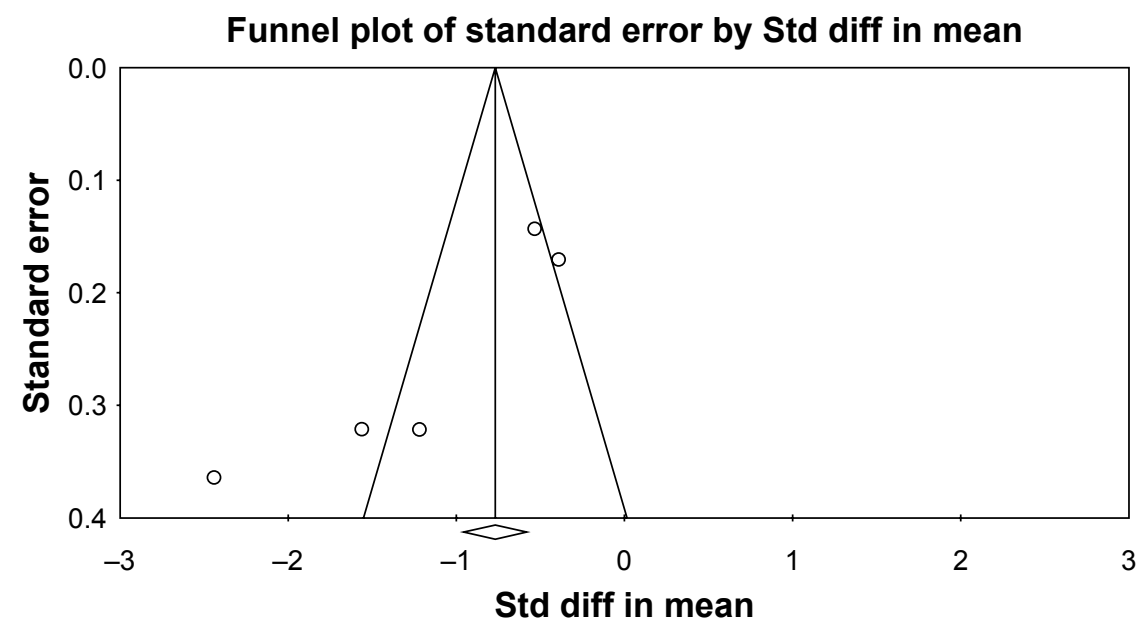

Figure 3 Funnel plot of effect sizes for the comparisons on global QOL.

Abbreviations: QOL, quality of life; Std diff, standard difference.

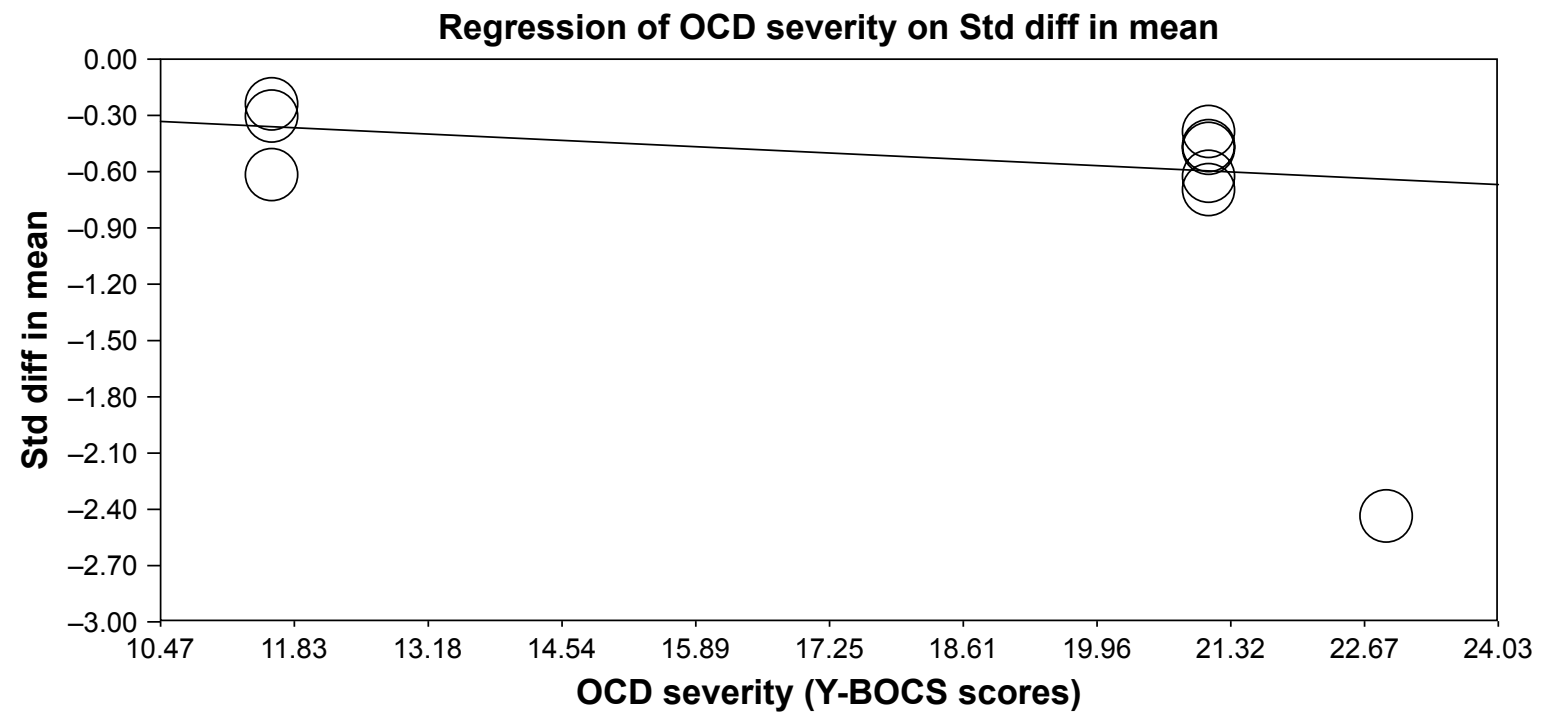

Figure 4 Meta-regression of global $\mathrm{QOL}$ as a function of $\mathrm{OCD}$ severity.

Abbreviations: QOL, quality of life; OCD, obsessive-compulsive disorder; Std diff, standard difference; Y-BOCS, Yale-Brown Obsessive Compulsive Scale. 


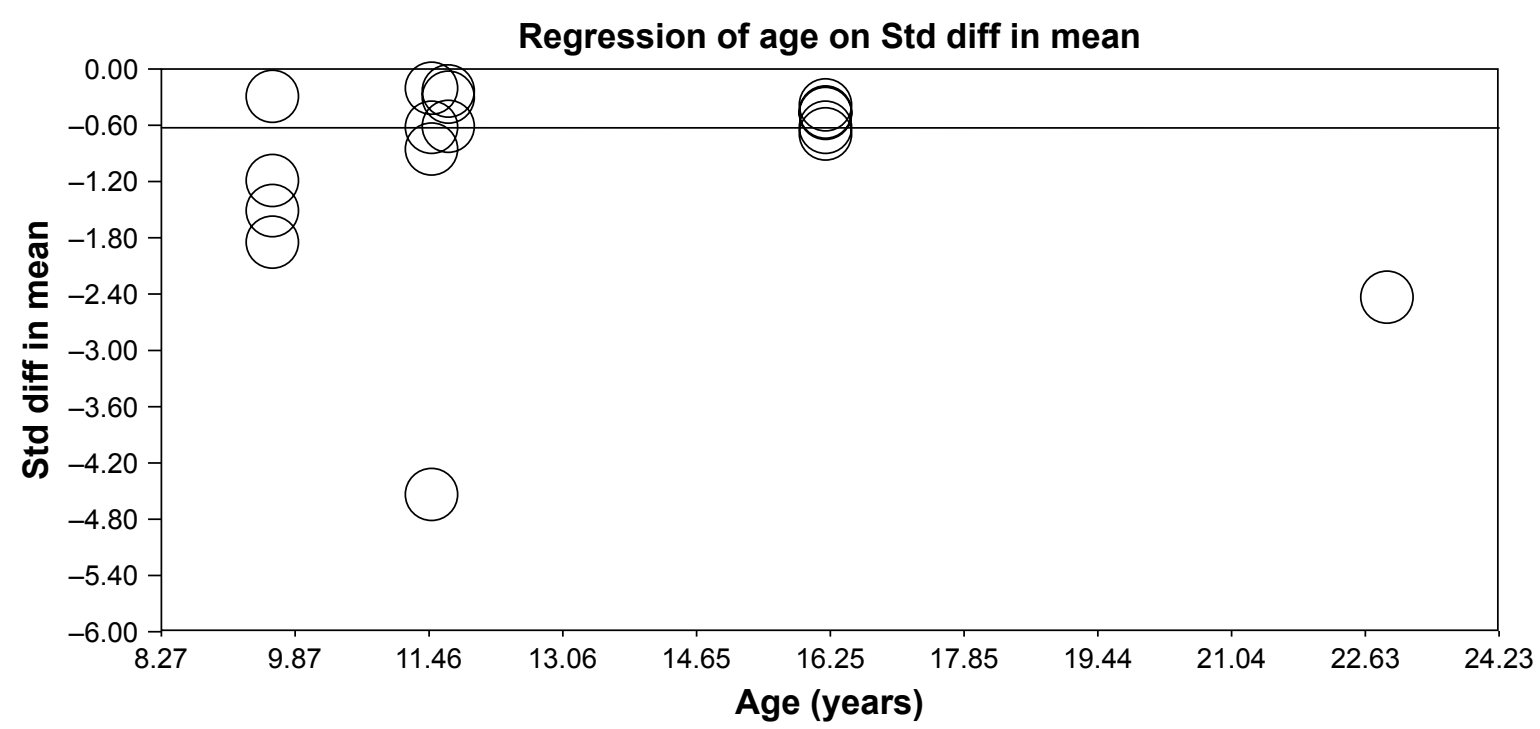

Figure 5 Meta-regression of global $\mathrm{QOL}$ as a function of age.

Abbreviations: QOL, quality of life; Std diff, standard difference.

Figure 10. A summary of effect sizes for all the analyses is presented in Table 3.

\section{Discussion}

QOL in children and adolescents with OCD is an understudied topic. Investigating QOL is an important issue for assessment and treatment, as the patient may present with reduced symptoms that do not necessarily translate into better QOL. Knowledge about QOL dimensions among children and adolescents suffering from this disorder can inform practice and research about the use of QOL outcomes in trials of pharmacotherapy and psychotherapy. ${ }^{8}$ This study was the first meta-analysis summarizing differences in QOL among children/adolescents with OCD and controls, specifically in global, social and school-related QOL dimensions. After an electronic search, five case-control studies were included with 543 participants. Findings indicated a large effect size without publication bias, suggesting that individuals with OCD had significantly lower global QOL than controls. In addition, moderate effect sizes were also observed for social and school-related QOL. Subsequently, analyses of moderators showed a negative relation between OCD severity and effect sizes, highlighting that for samples with more severe OCD symptoms, QOL was lower than

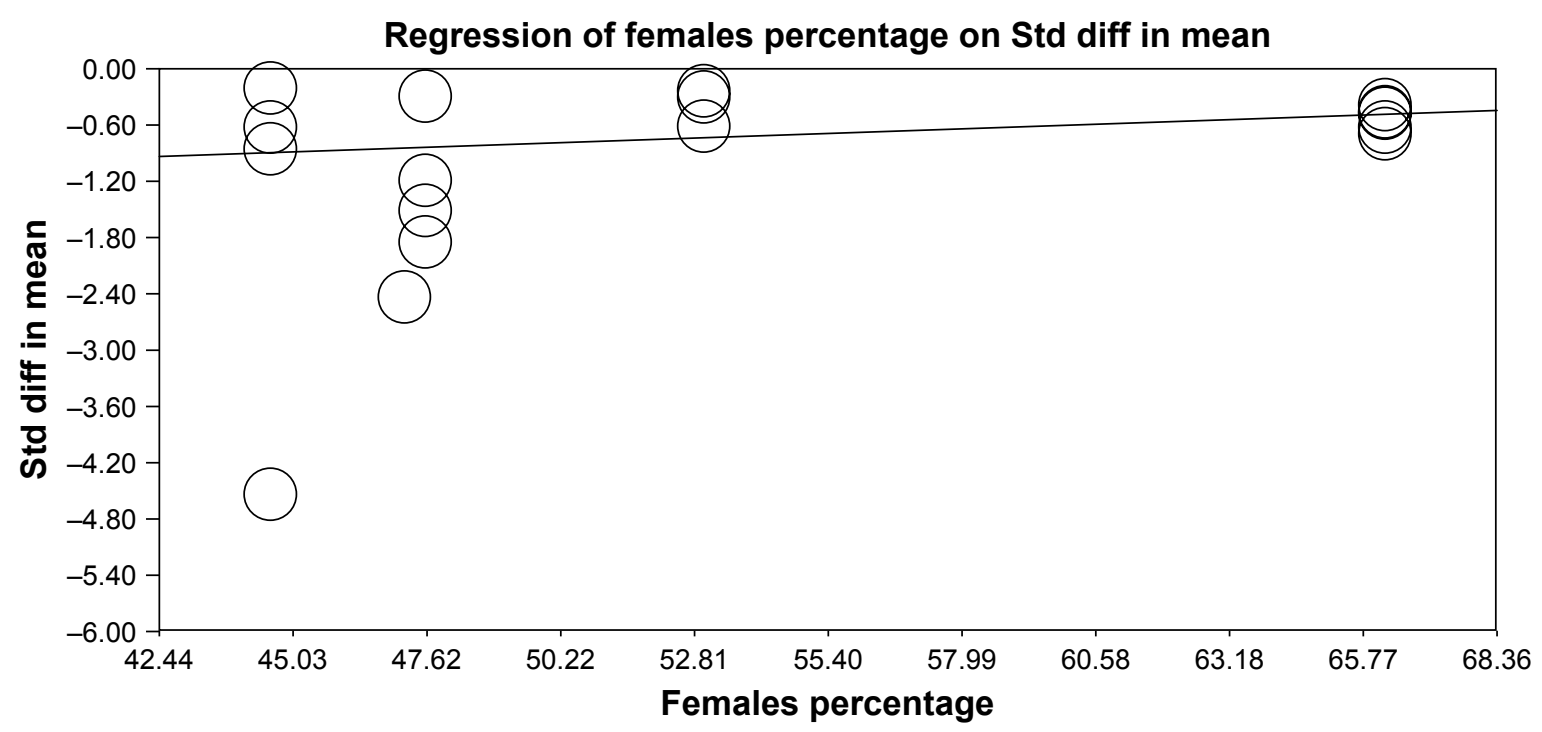

Figure 6 Meta-regression of global QOL as a function of sex. Abbreviations: QOL, quality of life; Std diff, standard difference. 
Table 2 Summary of moderators' analysis of effect sizes on global QOL

\begin{tabular}{lll}
\hline Moderators & $\boldsymbol{\beta}(\boldsymbol{P}$-value $)$ & $\mathbf{9 5 \%} \mathbf{C l}$ \\
\hline OCD severity (CY-BOCS scores) & $-0.02(<0.05)$ & -0.05 to $0.00 \mathrm{I}$ \\
Age (years) & $0.01(0.99)$ & -0.03 to 0.03 \\
Sex & $0.02(<0.00 \mathrm{I})$ & 0.02 to $0.0 \mathrm{I}$ \\
\hline
\end{tabular}

Abbreviations: $\mathrm{Cl}$, confidence interval; $\mathrm{CY}$-BOCS, Children's Yale Brown Obsessive Compulsive Scale; OCD, obsessive-compulsive disorder; QOL, quality of life.

that of controls. This result was in contrast with some previous knowledge in the literature indicating that $\mathrm{OCD}$ among children/adolescents is typically characterized by the absence of insight of symptoms and the associated impairment. ${ }^{7}$ Results of the previous study of De Caluwé and De Clercq ${ }^{7}$ indicated that OCD symptoms were not a significant predictor of QOL when impairment was included in the regression model. This inconsistency with our findings could be attributed to the fact that the study of De Caluwe and De Clercq ${ }^{7}$ did not use a case-control design, instead investigated the role of OCD symptoms and impairment as predictors of QOL in a large sample of community youth with OCD without including a clinical group. Another explanation could be the use of a father-rated measure of QOL and new measure of OCD symptoms in that study, ${ }^{7}$ the Youth Obsessive-Compulsive Symptoms Scale (YOCSS), ${ }^{29}$ a self-report scale assessing both youth OCD symptomatology and OCD impairment. In addition, these findings were not consistent with research data on adults, which showed that QOL was lower among individuals with less severe OCD. ${ }^{11}$ This finding pointed out the introduction of strategies to improve QOL for young individuals with more severe symptoms along with treatments focusing on the reduction of symptomatology. For example, family-based cognitive behavioral therapy could be treatment strategy able to improve also family QOL and functioning. Some recent randomized controlled trials ${ }^{30}$ showed that CBT with parents' involvement, where exposure and response prevention are enhanced by a parent, can be effective for reducing child/ adolescent OCD.

Sex could have a crucial role in moderating the effects of OCD on QOL. Current findings showed that male sex was associated with lower QOL. These data were in contrast with that found previously on adults, which suggested that female sex was associated with lower QOL. ${ }^{11}$ A possible explanation for this difference could be related to other studies on youth, which instead found that among individuals with OCD, males had more severe characteristics, including earlier age of onset. ${ }^{13}$ It could be hypothesized that differences in QOL between males and females corresponded to differences in the clinical picture of OCD symptoms.

With regard to age, the relation with effect sizes was not significant. The findings of this study summarized data on this relation, as previous data were inconsistent: some studies reported differences in the clinical picture, including severity and QOL impairment, between children and adolescents and others not. ${ }^{4,12}$ Moreover, according to other previous studies, young individuals with older age had more comorbid mood disorders, ${ }^{26}$ whereas other studies showed that younger individuals had stronger separation anxiety and tic disorders than the older ones. ${ }^{17}$ The absence of a relation between QOL and age found in the present meta-analysis was in line with the current literature, indicating that symptom patterns do not change across children and adolescents groups. ${ }^{4}$

Finally, some important limitations should be considered. First, the small number of included studies highlighted that the topic is still under-studied. The low number of studies was a main limitation that leads to low statistical power in the analyses, particularly when moderators were

\begin{tabular}{|c|c|c|c|c|c|c|c|c|c|c|c|c|c|c|c|c|c|}
\hline \multirow[t]{2}{*}{ Study name } & \multirow[t]{2}{*}{ Country } & \multirow[t]{2}{*}{ Comparison } & \multirow[t]{2}{*}{ Outcome } & \multicolumn{7}{|c|}{ Statistics for each study } & \multicolumn{3}{|c|}{ Sample size } & \multicolumn{4}{|c|}{ Std diff in mean and $95 \% \mathrm{Cl}$} \\
\hline & & & & $\begin{array}{l}\text { Std diff } \\
\text { in mean }\end{array}$ & $\begin{array}{l}\text { Standard } \\
\text { error }\end{array}$ & Variance & $\begin{array}{l}\text { Lower } \\
\text { limit }\end{array}$ & $\begin{array}{l}\text { Upper } \\
\text { limit }\end{array}$ & Z-value & $P$-value & $\begin{array}{l}\text { OCD } \\
\text { patients }\end{array}$ & Controls & Total & & & & \\
\hline Sukhodolsky et al ${ }^{26}$ & USA & $\begin{array}{l}\text { Screened } \\
\text { controls }\end{array}$ & CBCL social & -0.306 & 0.170 & 0.029 & -0.639 & 0.027 & -1.800 & 0.072 & 56 & 93 & 149 & & & & \\
\hline Souza Vivan et $\mathrm{al}^{27}$ & Brasile & $\begin{array}{l}\text { Screened } \\
\text { controls }\end{array}$ & $\begin{array}{l}\text { WHOQOL- } \\
\text { BREF social }\end{array}$ & -0.388 & 0.143 & 0.020 & -0.667 & -0.108 & -2.720 & 0.007 & 75 & 150 & 225 & & & & \\
\hline Geller et al ${ }^{6}$ & USA & $\begin{array}{l}\text { Screened } \\
\text { controls }\end{array}$ & CBCL social & -0.627 & 0.254 & 0.065 & -1.125 & -0.129 & -2.467 & 0.014 & 33 & 32 & 65 & & & & \\
\hline \multirow[t]{4}{*}{ Kim et al ${ }^{25}$} & USA & $\begin{array}{l}\text { Screened } \\
\text { controls }\end{array}$ & CBCL social & -1.195 & 0.316 & 0.100 & -1.814 & -0.575 & -3.780 & 0.000 & 33 & 18 & 51 & & & & \\
\hline & & & & -0.543 & 0.157 & 0.025 & -0.850 & -0.235 & -3.463 & 0.001 & 197 & 293 & 490 & & & & \\
\hline & & & & & & & & & & & & & \multirow[t]{2}{*}{-8.00} & -4.00 & 0.00 & 4.00 & 8.00 \\
\hline & & & & & & & & & & & & & & Favors OCD patients & & Favors controls & \\
\hline
\end{tabular}

Meta-analysis

Figure 7 Forest plot of the comparisons on social QOL between individuals with OCD and controls.

Abbreviations: QOL, quality of life; OCD, obsessive-compulsive disorder; Std diff, standard difference; Cl, confidence interval; CBCL, Child Behavior Checklist; WHOQOLBREF, World Health Organization Quality of Life Instrument. 


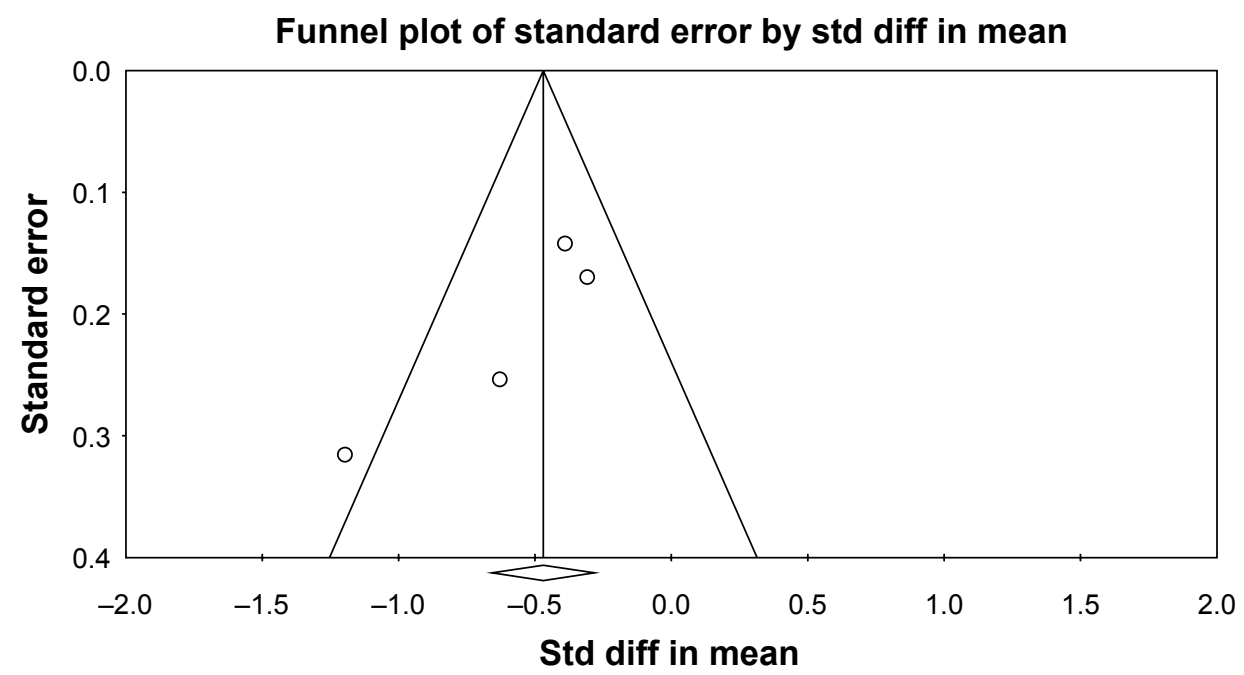

Figure 8 Funnel plot of effect sizes of comparisons on social QOL.

Abbreviations: QOL, quality of life; Std diff, standard difference.

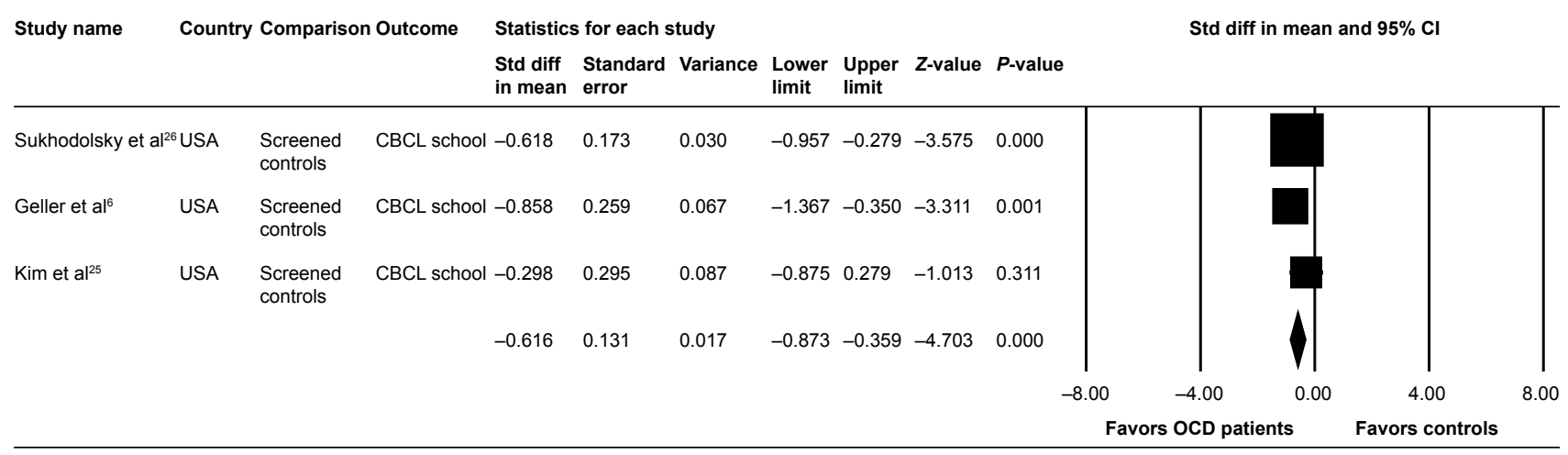

Meta-analysis

Figure 9 Forest plot of the comparisons on school QOL between individuals with OCD and controls.

Abbreviations: QOL, quality of life; OCD, obsessive-compulsive disorder; Std diff, standard difference; $\mathrm{Cl}$, confidence interval; $\mathrm{CBCL}$, Child Behavior Checklist.

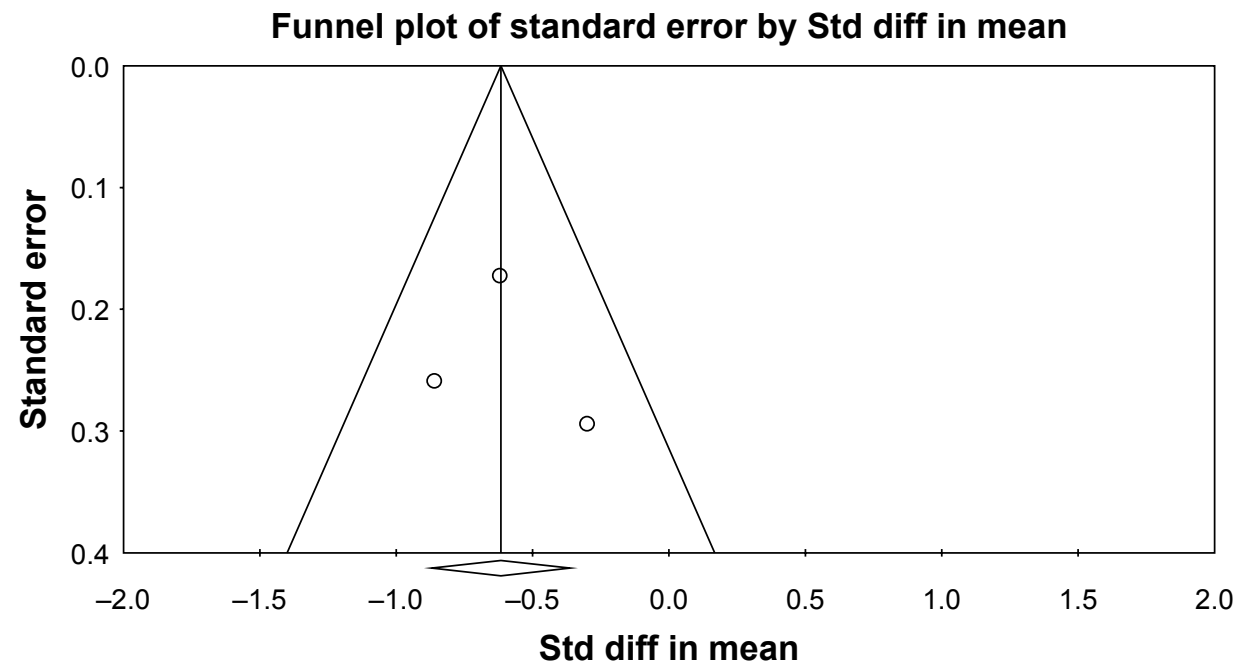

Figure I0 Funnel plot of the effect sizes on school QOL.

Abbreviations: QOL, quality of life; Std diff, standard difference. 
Table 3 Summary of effect sizes comparing children/adolescents with OCD with controls on QOL

\begin{tabular}{|c|c|c|c|c|c|c|c|c|}
\hline Comparisons & Outcomes & $k$ & $\bar{n}$ & $d$ (P-value) & $95 \% \mathrm{Cl}$ & $\mathrm{Q}$ (P-value) & $I^{2}$ & $\begin{array}{l}\text { Evidence of } \\
\text { publication bias }\end{array}$ \\
\hline $\begin{array}{l}\text { Children/adolescent patients } \\
\text { with OCD and controls }\end{array}$ & Global QOL & 5 & 543 & $-1.17(<0.001)$ & -1.79 to 0.54 & $36.6 I(<0.00 I)$ & 89.07 & Not found \\
\hline $\begin{array}{l}\text { Children/adolescent patients } \\
\text { with } O C D \text { and controls }\end{array}$ & Social QOL & 4 & 490 & $-0.54(<0.01)$ & -0.85 to 0.23 & $6.89(0.08)$ & 56.51 & Not found \\
\hline $\begin{array}{l}\text { Children/adolescent patients } \\
\text { with } O C D \text { and controls }\end{array}$ & $\begin{array}{l}\text { QOL related to } \\
\text { school functioning }\end{array}$ & 3 & 265 & $-0.6 \mathrm{I}(<0.0 \mathrm{I})$ & -0.87 to 0.36 & $2.04(0.36)$ & 1.89 & Not found \\
\hline
\end{tabular}

Abbreviations: $\mathrm{Cl}$, confidence interval; $\mathrm{OCD}$, obsessive-compulsive disorder; $\mathrm{QOL}$, quality of life.

tested. Consequently, the findings of this study were on preliminary evidence and suggested further studies. In this meta-analysis, studies comparing individuals with $\mathrm{OCD}$ and those with other psychiatric disorders were not included. It could be argued that the effects of OCD on QOL were not specific; instead, it could be attributable to a general effect of having psychopathological features. Otherwise, it could be interesting to evaluate whether specific domains of QOL are impaired among individuals with OCD relative to individuals presenting with other psychiatric diagnoses. Thus, further studies are required investigating QOL dimensions affected in OCD relative to other psychiatric disorders, such as schizophrenia and bipolar disorders. In addition, most of the studies did not report information about other important variables, which could be related to QOL in OCD, such as insight, OCD symptoms duration and family accommodation. ${ }^{10}$ Therefore, further studies investigating the role of these variables appear necessary. Since this meta-analysis included only case-control studies, future prospective studies are required to investigate whether these clinical variables could affect QOL outcomes over time. Another limitation was the use of parent- and patient-reported measures due to the low number of studies using only patient-reported measures. Thus, additional studies are required using patient-reported measures of QOL. Additional limitations concerned that other QOL outcomes, such as emotional QOL and QOL related to family functioning, were not included due a lack of studies investigating these aspects.

\section{Conclusion}

Preliminary findings from this meta-analysis encouraged researchers and clinicians to consider more carefully QOL dimensions and target them particularly for young males suffering from more severe OCD symptoms.

\section{Disclosure}

The authors report no conflicts of interest in this work.

\section{References}

1. American Psychiatric Association. Diagnostic and Statistical Manual of Mental Disorders (DSM5). 5th ed. Washington, DC: American Psychiatric Association; 2013.

2. Alvarenga PG, Cesar RC, Leckman JF, et al. Obsessive-compulsive symptom dimensions in a population-based, cross-sectional sample of school-aged children. J Psychiatr Res. 2015;62(3):108-114.

3. Zohar AH. The epidemiology of obsessive-compulsive disorder in children and adolescents. Child Adolesc Psychiatr Clin N Am. 1999; 8(3):445-460.

4. Hanna GL. Demographic and clinical features of obsessive-compulsive disorder in children and adolescents. $\mathrm{J}$ Am Acad Child Adolesc Psychiatry. 1995;34(1):19-27.

5. Ford T, Goodman R, Meltzer H. The British child and adolescent mental health survey 1999: the prevalence of DSM-IV disorders. J Am Acad Child Adolesc Psychiatry. 2003;42(10):1203-1211.

6. Geller DA, Biederman J, Faraone S, et al. Developmental aspects of obsessive-compulsive disorder: findings in children, adolescents, and adults. J Nerv Ment Dis. 2001;189(7):471-477.

7. De Caluwé E, De Clercq B. Obsessive-compulsive symptoms in children and adolescents: symptomatology, impairment and quality of life. Eur Child Adolesc Psychiatry. 2015;24(11):1389-1398.

8. Weidle B, Ivarsson T, Thomsen PH, Lydersen S, Jozefiak T. Quality of life in children with OCD before and after treatment. Eur Child Adolesc Psychiatry. 2015;24(9):1061-1074.

9. Tolin DF, Abramowitz JS, Diefenbach GJ. Defining response in clinical trials for obsessive-compulsive disorder: a signal detection analysis of the Yale-Brown Obsessive-Compulsive Scale. J Clin Psychiatry. 2005; 66(12):1549-1557.

10. Norberg MM, Calamari JE, Cohen RJ, Riemann BC. Quality of life in obsessive-compulsive disorder: an evaluation of impairment and a preliminary analysis of the ameliorating effects of treatment. Depress Anxiety. 2008;25(3):248-259.

11. Hollander E, Stein DJ, Fineberg NA, Marteau F, Legault M. Quality of life outcomes in patients with obsessive-compulsive disorder: relationship to treatment response and symptom relapse. J Clin Psychiatry. 2010;71(6):784.

12. Vasudev RGN, Yallappa SC, Saya GK. Assessment of quality of life (QOL) in obsessive compulsive disorder (OCD) and dysthymic disorder (DD): a comparative study. J Clin Diagn Res. 2015;9(5):4-7.

13. Masellis M, Rector MA, Richter MA. Quality of life in OCD: differential impact of obsessions, compulsions, and depression comorbidity. Can J Psychiatry. 2002;48(2):72-77.

14. Eisen JL, Mancebo MA, Pinto A, et al. Impact of obsessive-compulsive disorder on quality of life. Compr Psychiatry. 2006;47(4): 270-275.

15. Coluccia A, Fagiolini A, Ferretti F, et al. Adult obsessive-compulsive disorder and quality of life outcomes: a systematic review and metaanalysis. Asian J Psychiatr. 2016;22(8):41-52.

16. Mancebo MC, Garcia AM, Pinto A, et al. Juvenile-onset OCD: clinical features in children, adolescents and adults. Acta Psychiatr Scand. 2008;118(2):149-159. 
17. Shamseer L, Moher D, Clarke M, et al. Preferred reporting items for systematic review and meta-analysis protocols (PRISMA-P) 2015: elaboration and explanation. BMJ. 2015;349:g7647.

18. Kaufman J, Birmaher B, Brent D, et al. Schedule for affective disorders and schizophrenia for school-age children-present and lifetime version (K-SADS-PL): initial reliability and validity data. J Am Acad Child Adolesc Psychiatry. 1997;36(7):980-988.

19. Bernstein GA, Victor AM, Pipal AJ, Williams KA. Comparison of clinical characteristics of pediatric autoimmune neuropsychiatric disorders associated with streptococcal infections and childhood obsessivecompulsive disorder. J Child Adolesc Psychopharmacol. 2010;20(4): 333-340.

20. Scahill L, Riddle MA, McSwiggin-Hardin M, et al. Children's YaleBrown obsessive compulsive scale: reliability and validity. $J$ Am Acad Child Adolesc Psychiatry. 1997;36(6):844-852.

21. Cohen J. Statistical Power Analysis for the Behavioral Sciences. New York, NY: Routledge; 1988.

22. Cronbach LJ. Coefficient alpha and the internal structure of tests. Psychometrika. 1951;16(3):297-334.

23. Higgins JPT, Thompson SG, Deeks JJ, Altman DG. Measuring inconsistency in meta-analyses. BMJ. 2003;327(7414):557-560.

24. Duval S, Tweedie R. Trimm and fill: a simple funnel-plot-based method of testing and adjusting for publication bias in meta-analysis. Biometrics. 2000;56(2):455-463.
25. Kim KL, Reynolds KC, Alfano CA. Social impairment in children with obsessive compulsive disorder: do comorbid problems of inattention and hyperactivity matter? J Obsess Compuls Relat Disord. 2012;1(4):228-233.

26. Sukhodolsky DG, do Rosario-Campos MC, Scahill L, et al. Adaptive, emotional, and family functioning of children with obsessive-compulsive disorder and comorbid attention deficit hyperactivity disorder. Am J Psychiatry. 2005;162(6):1125-1132.

27. Sousa Vivan ADS, Rodrigues L, Wendt G, Bicca MG, Braga DT, Cordioli AV. Obsessive-compulsive symptoms and obsessive-compulsive disorder in adolescents: a population-based study. Rev Bras Psiquiatr. 2014;36(2):111-118.

28. Borda T, Feinstein BA, Neziroglu F, Veccia T, Perez-Rivera R. Are children with obsessive-compulsive disorder at risk for problematic peer relationships? J Obsessive Compuls Relat Disord. 2013;2(4): 359-365.

29. De Caluwé E, De Clercq B. Development and validation of the youth obsessive-compulsive symptoms scale (YOCSS). Child Psychiatry Hum Dev. 2014;45(6):647-656.

30. Freeman J, Sapyta J, Garcia A, et al. Family-based treatment of early childhood obsessive-compulsive disorder: the Pediatric ObsessiveCompulsive Disorder Treatment Study for Young Children (POTS Jr) a randomized clinical trial. JAMA Psychiatry. 2014;71(6):689-698.
Neuropsychiatric Disease and Treatment

\section{Publish your work in this journal}

Neuropsychiatric Disease and Treatment is an international, peerreviewed journal of clinical therapeutics and pharmacology focusing on concise rapid reporting of clinical or pre-clinical studies on a range of neuropsychiatric and neurological disorders. This journal is indexed on PubMed Central, the 'PsycINFO' database and CAS,

\section{Dovepress}

and is the official journal of The International Neuropsychiatric Association (INA). The manuscript management system is completely online and includes a very quick and fair peer-review system, which is all easy to use. Visit http://www.dovepress.com/testimonials.php to read real quotes from published authors. 